Submitted to THE ApJ

Preprint typeset using $\mathrm{LAT}_{\mathrm{E}} \mathrm{X}$ style emulateapj v. 5/2/11

\title{
A POPULATION OF RELIC INTERMEDIATE-MASS BLACK HOLES IN THE HALO OF THE MILKY WAY
}

\author{
VALERY RashKov AND PiERo MADAU \\ Department of Astronomy and Astrophysics, University of California, Santa Cruz, 1156 High Street, Santa Cruz, CA 95064. \\ submitted to the ApJ
}

\begin{abstract}
If "seed" central black holes were common in the subgalactic building blocks that merged to form present-day massive galaxies, then relic intermediate-mass black holes (IMBHs) should be present in the Galactic bulge and halo. We use a particle tagging technique to dynamically populate the N-body Via Lactea II high-resolution simulation with black holes, and assess the size, properties, and detectability of the leftover population. The method assigns a black hole to the most tightly bound central particle of each subhalo at infall according to an extrapolation of the $M_{\mathrm{BH}}-\sigma_{*}$ relation, and self-consistently follows the accretion and disruption of Milky Way progenitor dwarfs and their holes in a cosmological "live" host from high redshift to today. We show that, depending on the minimum stellar velocity dispersion, $\sigma_{m}$, below which central black holes are assumed to be increasingly rare, as many as $\sim 2000\left(\sigma_{m}=3 \mathrm{~km} \mathrm{~s}^{-1}\right)$ or as few as $\sim 70\left(\sigma_{m}=12 \mathrm{kms}^{-1}\right)$ IMBHs may be left wandering in the halo of the Milky Way today. The fraction of IMBHs kicked out of their host by gravitational recoil is $\lesssim 20 \%$. We identify two main Galactic subpopulations, "naked" IMBHs, whose host subhalos were totally destroyed after infall, and "clothed" IMBHs residing in dark matter satellites that survived tidal stripping. Naked IMBHs typically constitute 40-50\% of the total and are more centrally concentrated. We show that, in the $\sigma_{m}=12 \mathrm{~km} \mathrm{~s}^{-1}$ scenario, the clusters of tightly bound stars that should accompany naked IMBHs would be fainter than $m_{V}=16$ mag, spatially resolvable, and have proper motions of 0.1-10 milliarcsec per year. Their detection may provide an observational tool to constrain the formation history of massive black holes in the early Universe.

Subject headings: black hole physics - Galaxy: halo - stellar content - galaxies: evolution - dwarf method: numerical
\end{abstract}

\section{INTRODUCTION}

Direct dynamical measurements show that most local massive galaxies host a quiescent massive black hole $(\mathrm{MBH})$ in their nuclei. Their masses have been found to correlate tightly with the mass (Häring \& Rix 2004) and the stellar velocity dispersion of the host stellar bulge, as manifested in the $M_{\mathrm{BH}}-\sigma_{*}$ relation of spheroids (Ferrarese \& Merritt 2000; Gebhardt et al. 2000). It is not yet understood whether such scaling relations were set in primordial structures and maintained throughout cosmic time with a small dispersion, or indeed which physical processes established such correlations in the first place. It is also unclear whether there exists a minimum host galaxy mass or velocity dispersion below which MBHs are unable to form or grow. The subset of the MBHs that populates the centers of dwarf galaxies and very late spirals and is undergoing accretion can be detected as active galactic nuclei (AGNs). Observations of AGNs with estimated black hole masses in the range $10^{5}-10^{6} \mathrm{M}_{\odot}$ appear to be consistent with an extrapolation of the local $M_{\mathrm{BH}}-\sigma_{*}$ relation for inactive galaxies down to stellar velocity dispersions of $30 \mathrm{~km} \mathrm{~s}^{-1}$ (Barth et al. 2005; Xiao et al. 2011). The existence of "intermediate-mass" black holes (IMBHs) with $20 \mathrm{M}_{\odot}<M_{\mathrm{BH}}<10^{5} \mathrm{M}_{\odot}$ remains in dispute. Dynamical mass measurements have shown the presence of $\mathrm{IMBH}$ candidates in the cores of the globular clusters $\mathrm{G} 1\left(M_{\mathrm{BH}}=1.8 \pm 0.5 \times 10^{4} \mathrm{M}_{\odot}\right.$, Gebhardt et al. 2005), $\omega$ Centauri $\left(M_{\mathrm{BH}}=4.7 \pm 1.0 \times 10^{4} \mathrm{M}_{\odot}\right.$, Noyola et al. 2010), NGC 1904 and $6266\left(M_{\mathrm{BH}}=3 \pm 1 \times 10^{3} \mathrm{M}_{\odot}\right.$ and $M_{\mathrm{BH}}=2 \pm 1 \times 10^{3} \mathrm{M}_{\odot}$, Lützgendorf et al. 2012).
The X-ray spectra and bolometric luminosities of the ultraluminous off-nuclear X-ray sources detected in nearby galaxies may imply the presence of IMBHs with $M_{\mathrm{BH}} \gtrsim$ $500 \mathrm{M}_{\odot}$ (Farrell et al. 2009; Kaaret et al. 2001).

While the "seeds" of the MBHs powering the $z \gtrsim 6$ Sloan Digital Sky Survey (SDSS) very luminous, rare quasars (Fan 2006) must have appeared at very high redshifts and grown rapidly to more than $10^{9} \mathrm{M}_{\odot}$ in less than a Gyr, the problem of their origin and occupation fraction in early galaxies remains unsolved. MBHs may have grown from the remnants of Population III (Pop III) star formation in sub-dwarf galaxies at $z \gtrsim 15$ (e.g. Madau \& Rees 2001; Volonteri et al. 2003; Tanaka \& Haiman 2009), or from more massive precursors formed by the "direct collapse" of large amounts of gas in dwarf galaxy systems at later times (Loeb \& Rasio 1994; Koushiappas et al. 2004; Begelman et al. 2006; Lodato \& Natarajan 2006; Maver et al. 2010). Massive seeds have larger masses than Pop III remnants, but form in rarer hosts. Questions remain in the Pop III remnant model about the ability of $\sim 100 \mathrm{M}_{\odot}$ seed holes to grow at the Eddington rate for tens of e-folding times unimpeded by feedback, and in the direct collapse model about the needed large supply of low angular momentum gas that must accumulate in the center of young galaxies before fragmentation and star formation sets in (see, e.g., Haiman 2012 and references therein).

In this Paper we assess the size, properties, and detectability of the leftover population of $\mathrm{IMBH} \square$ that

1 In the following, we will use the term IMBH to refer to any hole with $20 \mathrm{M}_{\odot}<M_{\mathrm{BH}}<10^{5} \mathrm{M}_{\odot}$, i.e. more massive than the 
is predicted to survive today in the halo of the Milky Way (MW) galaxy by two fiducial seeding scenarios. It was first pointed out by Madau \& Rees (2001) that, if seed holes were indeed common in the subgalactic building blocks that merged to form the present-day massive galaxies, then a numerous population of relic IMBHs should be present in the Galactic bulge and halo (see also Volonteri et al. 2003; Islam et al. 2003; Volonteri \& Perna 2005). Micic et al. (2006, 2011) used collisionless simulations to study the effect of gravitational recoil kicks on the IMBH distribution in presentday galaxies and test merger-driven recipes for black hole growth. van Wassenhove et al. (2010) ran Monte Carlo realizations of the merger history of massive galaxy halos to study IMBHs in MW satellites at $z=0$. A similar approach was employed by O'Leary \& Loeb (2009) to estimate the expected number of recoiled IMBH remnants present today in the MW halo. Cosmological hydrodynamic simulations of the growth of MBHs are either limited by resolution to galaxy hosts with $M_{\text {halo }} \gtrsim 10^{10} \mathrm{M}_{\odot}$ (e.g. Di Matteo et al. 2008; Dubois et al. 2012) or stop at high redshift (e.g. Bellovary et al. 2011). In all approaches seed holes are planted following prescriptions that are based either on local properties such as gas angular momentum, temperature, metallicity, etc, or on global properties such as the mass or circular velocity of the host halo. To complement the above calculations, we use here a particle tagging technique to dynamically populate the N-body Via Lactea II (VLII) extremeresolution simulation with IMBHs. As we shall discuss, this method allows us to self-consistently follow the accretion and disruption of thousands of MW progenitor dwarfs and the kinematics of their holes in a cosmological "live" host, and the build-up of a large population of Galactic "naked" wandering IMBHs.

\section{BLACK HOLE TAGGING TECHNIQUE}

The cosmological $\Lambda$ CDM VLII simulation, one of the highest-precision N-body calculations of the assembly of the Galactic halo to date (Diemand et al. 2008), was performed with the PKDGRAV tree-code (Stadel 2001). It employs just over one billion $4,100 \mathrm{M}_{\odot}$ particles to model the formation of a $M_{200}=1.93 \times 10^{12} \mathrm{M}_{\odot}$ Milky Way-sized halo and its substructure. About 20,000 surviving subhalos of masses above $10^{6} \mathrm{M}_{\odot}$ are resolved today within the main host's $r_{200}=402 \mathrm{kpc}$ (the radius enclosing an average density 200 times the mean matter value). Central black holes are added to subhalos following the particle tagging technique detailed in Rashkov et al. (2012) and quickly summarized here. In each of 27 (out of the 400 available) snapshots of the simulation, chosen to span the assembly history of the host between redshift $z=27.54$ and the present, all subhalos are identified and linked from snapshot to snapshot to their most massive progenitor: the subhalo tracks built in this way contain all the time-dependent structural information necessary for our study. We then: 1) identify the simulation snapshot in which each subhalo reaches its maximum mass, $M_{\text {halo }}$, before being accreted by the main host and tidally stripped; 2) measure the subhalo maximum circular velocity $\left.V_{\max } ; 3\right)$ link it to the stel-

stellar-mass black holes found in X-ray emitting binary systems (Orosz et al. 2007) and 40 times less massive than Sgr A*. lar line-of-sight velocity dispersion, $\sigma_{*}$, using the relation $V_{\max }=2.2 \sigma_{*}$ derived by Rashkov et al. (2012); and 4) tag the most tightly bound central particle as a black hole of mass $M_{\mathrm{BH}}$ according to an extrapolation of the $M_{\mathrm{BH}}-\sigma_{*}$ relation of Tremaine et al. (2002),

$$
\frac{M_{\mathrm{BH}}}{\mathrm{M}_{\odot}}= \begin{cases}10^{6.91}\left(\frac{\sigma_{*}}{100 \mathrm{~km} \mathrm{~s}^{-1}}\right)^{4} & \left(\sigma_{*} \geq 6 \mathrm{~km} \mathrm{~s}^{-1}\right) \\ 100 & \left(\sigma_{*}<6 \mathrm{~km} \mathrm{~s}^{-1}\right) .\end{cases}
$$

A significantly steeper $M_{\mathrm{BH}}-\sigma_{*}$ relations have been derived recently by, e.g. Graham et al. (2011) and McConnell \& Ma (2013), and we will discuss the impact of such a steeper power-law on our results in $\S 6$. By neglecting all the poorly resolved subhalos with $M_{\text {halo }}<10^{7} \mathrm{M}_{\odot}$, we restrict our analysis to $3,204 \mathrm{such}$ tracks. Note that, with such a cut, about 200 subhalos below $10^{7} \mathrm{M}_{\odot}$ and with $\sigma_{*}>3 \mathrm{~km} \mathrm{~s}^{-1}$ (equivalent to $V_{c}>6.6 \mathrm{kms}^{-1}$ ) are not actually assigned a central black hole. These "missed" IMBHs account, however, for less than $10 \%$ of all possible IMBHs that are tagged at these low stellar velocity dispersions.

Any evolution of the tagged holes after infall is purely kinematical in character, as their satellite hosts are accreted and disrupted in an evolving Milky Way-sized halo. After tagging, the black hole particles are tracked down to the $z=0$ snapshot. The main host is assigned a central black hole at $z=0$ of mass equal to that of Sgr A*, $M_{\mathrm{BH}}=4 \times 10^{6} \mathrm{M}_{\odot}$ (Ghez et al. 2008). While we tag at most one hole per subhalo, we allow multiple systems to form when subhalos merge after infall (see below). Figure 1 shows the distributions of stellar velocity dispersion at infall (left panel) and time since infall (right panel) for the VLII subhalo population. The color coding separates self-gravitating subhalos that survive their accretion event and become MW satellites from those that are totally disrupted. The latter are found to fall in preferentially at earlier times, and have a median infall redshift of 4.5 .

\section{DEMOGRAPHY OF IMBHS}

Below we discuss two simple models of seed hole formation that may be illustrative of more realistic growth scenarios, and differ only in the black hole occupation fraction as a function of the host's stellar velocity dispersion.

\subsection{Population III Remnants}

Let us first consider a scenario where the black hole occupation fraction is of order unity at infall in all subhalos with stellar velocity dispersions $\geq \sigma_{m}=3 \mathrm{~km} \mathrm{~s}^{-1}$ and drops to zero below $\sigma_{m}$. This value of $\sigma_{m}$ is comparable to the stellar velocity dispersion measured today in the ultra-faint MW satellite Segue 1 (Simon et al. 2011), i.e. this model places seed holes in small-mass subhalos that are known to have been rather inefficient at forming stars (e.g. Rashkov et al. 2012; Cooper et al. 2010; Koposov et al. 2009). The mass distribution of subhalos with $\sigma_{*}>3 \mathrm{~km} \mathrm{~s}^{-1}$ at infall has a median of $5 \times 10^{7} \mathrm{M}_{\odot}$, so these subhalos are well resolved in our simulation. We assign black holes masses following the relation (1). As most of the subhalos in Fig. 1 host then a $100 \mathrm{M}_{\odot}$ black hole, we refer to this model as "Pop III remnants". We note that this scenario is not based on a specific physi- 

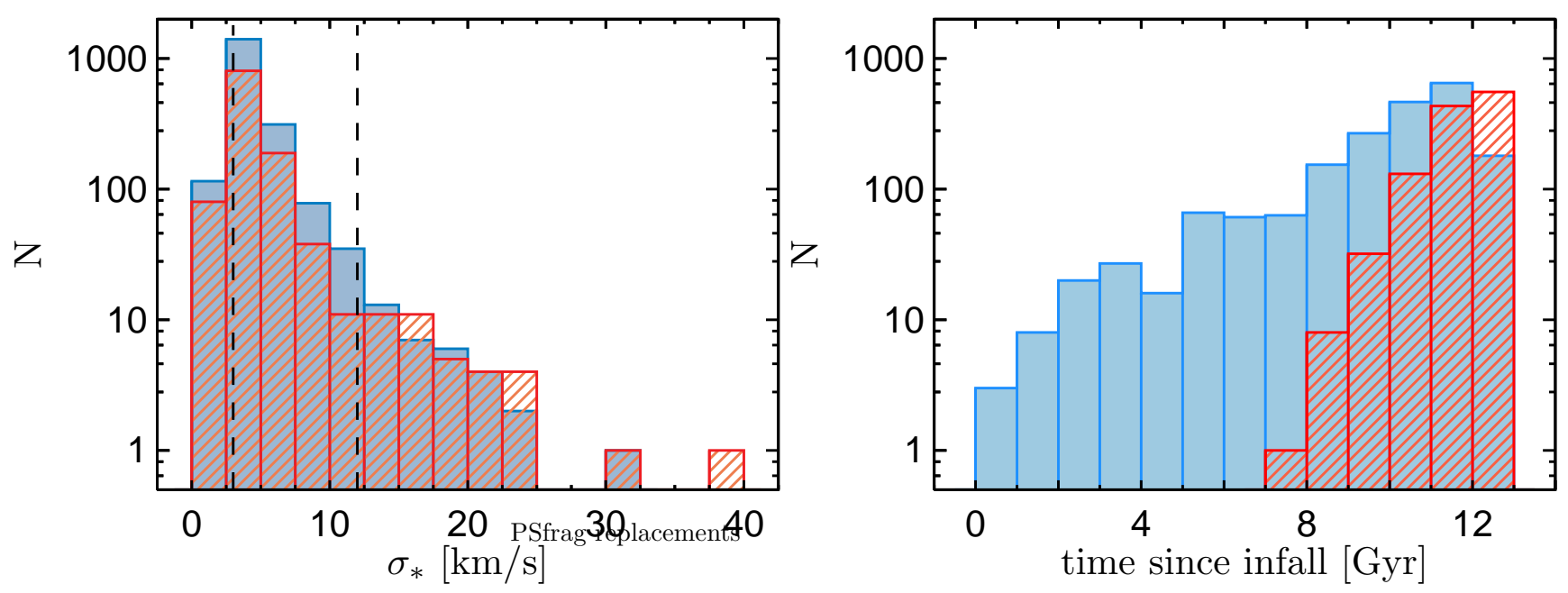

FIG. 1.- Histograms of the stellar velocity dispersion at infall (left panel) and infall lookback time (right panel) for all subhalos with $M_{\text {halo }}>10^{7} \mathrm{M}_{\odot}$ in the VLII simulation. The color coding separates self-gravitating subhalos that survive their accretion event and become MW satellites (blue) from those that are totally disrupted (red). The vertical lines in the left panel mark the minimum velocity dispersion below which central black holes are assumed to be increasingly rare in the two seeding scenarios discussed in the text.

cal model, but is simply meant to be used for comparison with existing and forthcoming numerical simulations. The occupation fraction is arbitrarily set to unity but can be easily scaled down to account for a reduced MBH formation efficiency on these small subhalo mass scales.

Two large subpopulations of Galactic black holes can then be readily identified today within $r_{200}, 798$ "naked" IMBHs, whose host subhalos have been totally disrupted after infall, and 1,234 "clothed" IMBHs residing in satellites that survived tidal stripping. The abundance of Galactic black holes in this model is comparable with the predictions of semi-analytical calculations by Islam et al. (2004) for Milky Way-sized halos (their Model A). These authors also identified a population of "naked" IMBHs following the tidal disruption of infalling satellites, but their estimated naked fraction appears smaller than we find in our simulations (see Fig. 2 of Islam et al. 2004). As expected, in both calculations (as well as in those of Volonteri \& Perna 2005) there are more naked holes near the center of the host, where tidal forces are stronger. Indeed, within $10 \mathrm{kpc}$ of the center, most IMBHs are naked.

Within the "clothed" category, 1,096 are single black hole systems, i.e. are located in subhalos that host only one hole at the present epoch. The others are multiple systems: we find 42 dwarf subhalos with 2 holes, 7 with 3,5 with 4 , and 1 with each of 6 and 7 holes. These multiple systems are produced when their host subhalos merge together after infall, as they fall into the main halo in groups. We assume that by that time, ram pressure stripping has removed any leftover gas from the system, making it difficult for IMBHs to sink to the center of the potential well and merge. Substructures that host 5 or more IMBHs are found to be more than $250 \mathrm{kpc}$ from the Galactic center and have masses in the range $2 \times 10^{9}-3 \times 10^{9} \mathrm{M}_{\odot}$ today. Figure2(left panels) shows an image of the projected spatial distribution of IMBHs in today's inner and outer Galactic halo according to this model, while Figure 3 depicts their mass function and radial distribution. Naked holes are more concentrated towards the inner halo regions as a consequence of the tidal disruption of infalling satellites. Indeed, within 10 kpc of the center, most IMBHs are naked. The second most massive hole (after "Sgr A*") has $M_{\mathrm{BH}}=8.6 \times$ $10^{4} \mathrm{M}_{\odot}$ (i.e. was tagged at infall in a large satellite with $\left.\sigma_{*}=32 \mathrm{~km} \mathrm{~s}^{-1}\right)$, is naked, and is located at a distance of $55 \mathrm{kpc}$ from the center. There are 48 IMBHs above $M_{\mathrm{BH}}=10^{3} \mathrm{M}_{\odot}$, of which 29 are naked. Most holes are assigned the seed mass of $100 \mathrm{M}_{\odot}$, i.e. they are not allowed to grow in this model: we count a total of 1,801 such "light" IMBHs, of which 695 are naked.

\subsection{Direct Collapse Precursors}

Consider by contrast a scenario where more massive seeds form in rarer hosts. Models in which seed black holes become very rare for halos with $M_{\text {halo }} \lesssim 10^{9} \mathrm{M}_{\odot}$ have been recently discussed by, e.g., Bellovary et al. (2011) and Devecchi et al. (2012). Here, we take the hole occupation fraction to be of order unity in all subhalos with stellar velocity dispersions $\geq \sigma_{m}=12 \mathrm{~km} \mathrm{~s}^{-1}$, and to drop to zero below $\sigma_{m}$. Black hole masses are again assigned following equation (1) down to $\sigma_{m}$. The mass distribution of subahalos hosting central black holes at infall now has a median of $3 \times 10^{9} \mathrm{M}_{\odot}$, and the minimum hole mass is $M_{\mathrm{BH}} \simeq 1,700 \mathrm{M}_{\odot}$. Again, this scenario is not based on a specific physical model but is simply meant to be illustrative of a situation in which dwarf spheroidal satellites of the Milky Way with stellar velocity dispersions comparable to that of Ursa Minor (see, e.g., Wolf et al. 2010 for a compilation) would actually host an IMBH. Recent observations have shown that dwarf galaxies with stellar masses comparable to the Magellanic Clouds do exhibit optical spectroscopic signatures of accreting MBHs (Reines et al. 2013). Note that, in the models of Bellovary et al. (2011), halos with virial masses above $3 \times 10^{9} \mathrm{M}_{\odot}$ are predicted to always host a MBH seed, regardless of the seed formation efficiency. This is because, even in the lowest efficiency case, MBH seeds form in the region of earliest star formation, which tend to be the halos that becomes the most massive later on in every simulation. Moreover, the most massive halos have also experienced the greatest num- 


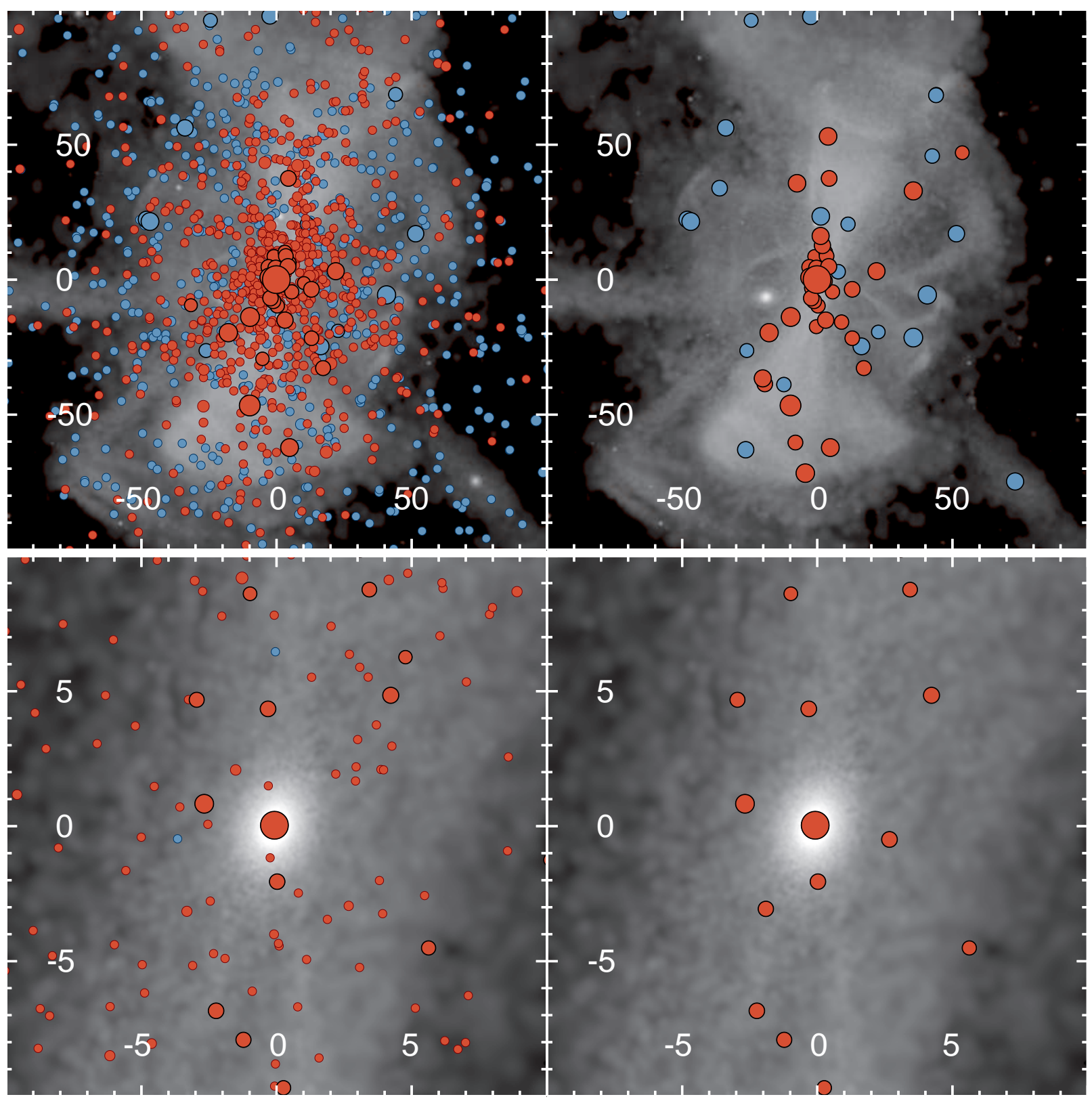

FIG. 2.- Projected distribution of IMBHs in the Galactic halo today. Left panels: Pop III remnant model. Right panels: Direct collapse progenitor model. The color coding is as in Fig. 1 while the size of the points is proportional to $\log _{10} M_{\mathrm{BH}}$. The stellar halo from Rashkov et al. (2012) is plotted in the background. Top row: $200 \mathrm{kpc}$ box. Bottom row: Zoom-in of the inner 20 kpc region.

ber of mergers, and are then populated by $\mathrm{MBH}$ seeds brought in by satellites.

The tagging prescription adopted in this scenario gives origin to 39 naked and 33 clothed IMBHs (of which 27 are single systems). Figure 2 (right panels) shows an image of the projected spatial distribution of IMBHs in today's inner and outer Galactic halo according to this model, while Figure 3 (left panel) depicts their mass function. There are $20 \mathrm{IMBH}$ above $M_{\mathrm{BH}}=10^{4} \mathrm{M}_{\odot}$, of which 13 are naked.

\section{BLACK HOLE RECOILS}

The asymmetric emission of gravitational waves produced during the coalescence of a binary black hole system imparts a velocity kick to the system that can dis- place the hole from the center of its host. The magnitude of the recoil will depend on the binary mass ratio and the direction and magnitude of their spins (Baker et al. 2008), but not on the total mass of the binary. When the kick velocity is larger than the escape speed of the host halo, a hole may be ejected into intergalactic space before becoming a Galactic IMBH. The demography of IMBHs discussed in the previous section accounts for recoiling holes as follows.

Following the results of high-resolution self-consistent gasdynamical simulations of binary mergers of disk galaxies by Kazantzidis et al. (2005), we assume that unequal-mass galaxy mergers with mass ratios larger than 4:1 will not lead to the formation of a close black 
hole pair at the center of the remnant, and hence of a recoiling hole. All major mergers (mass ratios smaller than 4:1) produce instead recoiling holes with a kick velocity that is equal to the most likely value of the probability distribution function for randomly oriented spins (see Fig. 3 of Guedes et al. 2011). In practice, this means that all recoiling holes will have speeds in excess of $100 \mathrm{~km} \mathrm{~s}^{-1}$ and will be ejected from the system. This is a conservative assumption in the sense that it tends to minimize the actual number of relic IMBHs in the Galactic halo. We trace all $<4: 1$ halo mergers following the VLII merger tree back to redshift 10 . This was built by identifying all subhalos in each snapshot with the 6DFOF halo finder (Diemand et al. 2006), and linking them from one snapshot to the next by the id numbers of the shared particles. We identify all halos that would have been assigned a hole at infall, but had in their past a $<4: 1$ major merger with another black hole-hosting system. As the kick velocity is always larger than the escape speed from the host, the hole is removed from the final catalog of Galactic IMBHs.

The size of the recoiling population is clearly very sensitive to the hole occupation fraction. Figure 4 shows the total number of halo major mergers that produce recoiling IMBHs in the "Pop III" and "direct collapse" seed scenarios. The former produces 577 recoiling holes $(22 \%$ of the total black hole population), mostly belonging to $\sim 10^{8} \mathrm{M}_{\odot}$ subhalos at infall. The latter produces only 5 recoiling holes ( $6 \%$ of the total) all from rather massive hosts. Note that it is because of recoils that the number of Galactic IMBHs heavier than a few thousand solar masses is actually larger in the direct collapse model (see Fig. 3). The demography of Galactic IMBHs in our two scenarios is summarized in Table 1 .

\section{SPATIAL DISTRIBUTION OF IMBHS}

The spatial distribution of Galactic IMBHs today is shown in Figure 5. Naked holes dominate in the inner $\sim$ $50 \mathrm{kpc}$ from the halo center. Their number density, however, plummets at larger distances, where most IMBHs are still hosted in surviving substructures. This is a manifestation of the fact that subhalos orbiting closer to the dense central regions of the main host are more likely to be tidally disrupted, and leave their IMBHs exposed. In the outer halo regions, the lower background density makes substructures more resilient to tidal forces, allowing them to survive and retain their holes. A comparison of the IMBH number density to the density profile of the dark matter shows that the cumulative distribution of Galactic IMBHs (naked plus clothed) follows the dark matter profile rather well, but that each individual subpopulation does not. The inset in Figure [5] shows that the IMBH mass density profile does not follow the density profile of the dark matter, as most of the mass in black holes is concentrated in the inner regions.

Since naked IMBHs more massive than dark matter particles do not experience proper dynamical friction in our simulation, we have estimated a posteriori the effect of black hole orbital decay in two different ways - using the slowly-decaying circular orbit approximation, as well as computing the instantaneous deceleration from dynamical friction (Binney \& Tremaine 2008) in each available VLII snapshot. We find that, even for the heaviest IMBHs in the highest density regions of our simu- lation, dynamical friction timescales exceed the Hubble time at all points along the orbit. A similar conclusion was reached by O'Leary \& Loeb (2009) in their study. Of course, our N-body simulation does not account for possible hydrodynamical effects and interactions with the galaxy stellar disk in the very center of the VLII halo.

\section{DISCUSSION}

We have used a particle tagging technique to populate the subgalactic building blocks of a present-day MWsized galaxy with black holes, and assess the size, properties, and detectability of the leftover population. Our approach combines a computationally expensive, extremeresolution, N-body simulation like VLII, in which structures grow ab initio in a fully cosmological framework, with simple prescriptions for seeding, in post-processing, progenitor subhalos with IMBHs. Insofar baryonic material does not appreciably perturb the collisionless dynamics of the N-body component, the dynamical association of black holes with individual particles in the N-body component should correctly reproduce the spatial and kinematic properties of IMBHs in galaxy halos, in particular of the naked subpopulation whose host satellite galaxies have been totally destroyed after infall. This level of detail is unavailable to a standard merger-tree approach.

We have discussed two simple models of seed hole formation that may be illustrative of more realistic growth scenarios, a "Pop III remnant" model in which smallmass black holes populate subhalos with stellar velocity dispersion as low as $3 \mathrm{~km} \mathrm{~s}^{-1}$, and a "direct collapse precursor" model in which holes become very rare in systems with stellar velocity dispersion below $12 \mathrm{~km} \mathrm{~s}^{-1}$, and are more massive. It is important to keep in mind that the Pop III route does not necessarily require the formation of extremely massive stars from gas of primordial composition. Already at metallicities $Z \lesssim 0.01 Z_{\odot}$, the mass loss rates of massive stars through radiatively driven stellar winds are predicted to be rather small (with rates decreasing with metallicity as $\dot{M} \propto Z^{0.69}$, see Vink et al. 2001). If low-metallicity stars above $50 \mathrm{M}_{\odot}$ collapse to black holes after losing only a small fraction of their initial mass, then IMBHs with masses above the $5-20 \mathrm{M}_{\odot}$ range of known "stellar-mass" holes may be the inevitable end product of early star formation. A standard Salpeter initial mass function (IMF), $d N / d m_{*} \propto m_{*}^{-2.35}$ in the range $1-100 \mathrm{M}_{\odot}$, would produce an IMBH every $1000 \mathrm{M}_{\odot}$ of stars formed. A top-heavy IMF like that found in recent simulations of primordial star formation by Stacy \& Bromm (2012), $d N / d m_{*} \propto m_{*}^{-0.17}$, over the same $1-100 \mathrm{M}_{\odot}$ mass range, would generate an IMBH every $100 \mathrm{M}_{\odot}$ of stars formed. Note that $1000 \mathrm{M}_{\odot}$ of stars in a $5 \times 10^{7} \mathrm{M}_{\odot}$ subhalo imply a star formation efficiency that is much too high to match the observed dearth of faint Milky Way satellites (e.g. Rashkov et al. 2012), and a top-heavy IMF must then be invoked for consistency.

Galactic IMBHs may light up if they pass through dense regions of the galaxy and accrete from the interstellar medium (Volonteri \& Perna 2005). IMBHs present in the lensing galaxy of multiply-imaged background QSOs will produce monopole- or dipole-like distortions in the surface brightness of the QSO images and may be detectable by next generation submillimeter telescopes with 

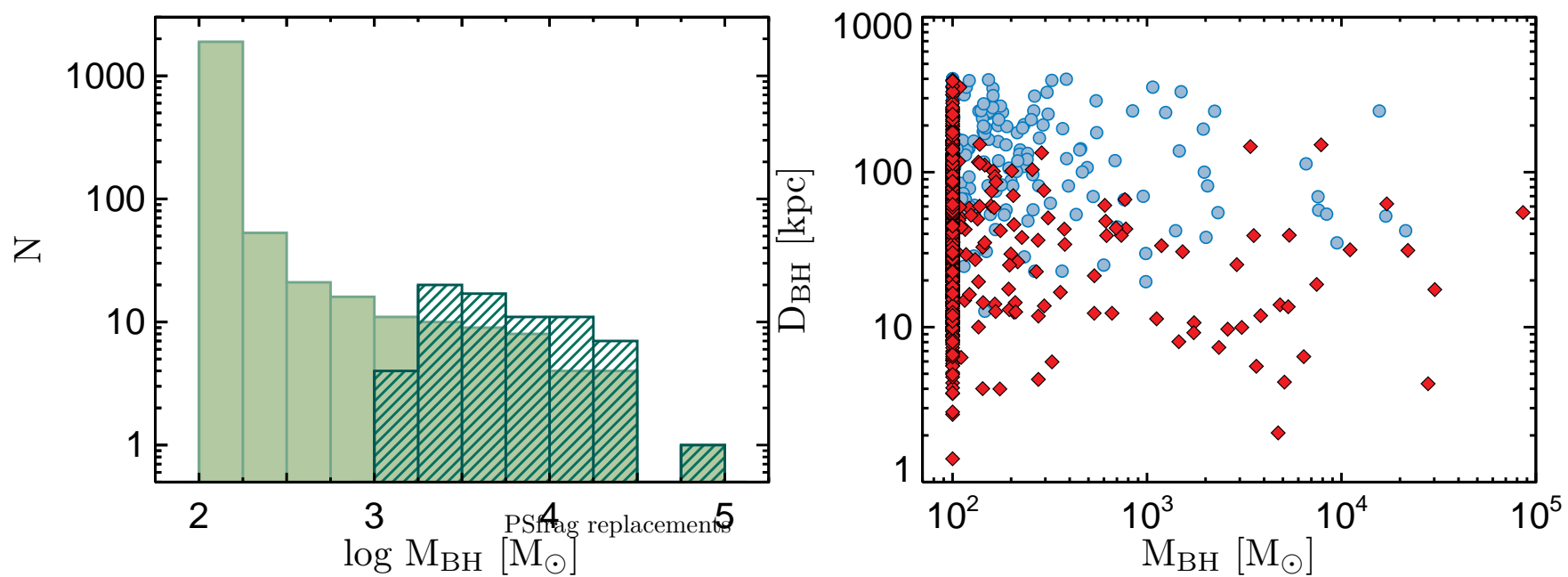

FIG. 3. - Left panel: Mass function of relic IMBHs within $r_{200}$ in the two scenarios discussed in the text. Solid histogram: Pop III remnants. Hatched histogram: Direct collapse precursors. Right panel: Location of "naked" (red diamonds) and "clothed" (blue circles) IMBHs in the $M_{\mathrm{BH}}$-Galactocentric distance plane for the Pop III model. Naked IMBHs are more concentrated towards the inner halo regions, a trend that is also observed in the direct collapse, massive seed scenario.

TABLE 1

Demography of Galactic IMBHs

\begin{tabular}{|c|c|c|c|c|c|c|c|c|}
\hline Model & $\begin{array}{c}\sigma_{m} \\
{\left[\mathrm{~km} \mathrm{~s}^{-1}\right]}\end{array}$ & $\begin{array}{c}\operatorname{med}\left(M_{\text {halo }}\right) \\
{\left[M_{\odot}\right]} \\
\end{array}$ & $N_{\text {naked }}$ & $N_{\text {clothed }}$ & $\begin{array}{c}N_{\text {massive }} \\
\left(M_{\mathrm{BH}}>5,000 \mathrm{M}_{\odot}\right)\end{array}$ & $\begin{array}{c}\min \left(M_{\mathrm{BH}}\right) \\
{\left[M_{\odot}\right]} \\
\end{array}$ & $\begin{array}{c}\max \left(M_{\mathrm{BH}}\right) \\
{\left[M_{\odot}\right]} \\
\end{array}$ & $\overline{N_{\text {recoiled }}}$ \\
\hline Popu & 3 & $5 \times 10^{7}$ & 798 & 1234 & 21 & 100 & $1.9 \times 10^{5}$ & 577 \\
\hline Direct Collapse & 12 & $3 \times 10^{9}$ & 39 & 33 & 37 & 1691 & $1.9 \times 10^{5}$ & 5 \\
\hline
\end{tabular}

Note. - Column 1 indicates the seeding scenario, columns $2,3,4,5,6,7,8$, and 9 give the minimum subhalo line-of-sight stellar velocity dispersion required for hosting a central black hole, the median halo mass at infall, the number of "naked" and "clothed" Galactic IMBHs, the number of Galactic IMBHs more massive than $5000 \mathrm{M}_{\odot}$, the mass of the lightest and heaviest Galactic IMBHs, and the number of recoiled IMBHs, respectively.

high angular resolution (Inoue et al. 2013). It is interesting at this stage to discuss the observability of the stellar cusps that may accompany naked IMBHs wandering in the Milky Way halo. After the complete disruption of their host subhalo, naked IMBHs are still expected to carry with them a Bahcall-Wolf stellar cusp (Merritt et al. 2009) which, upon formation and in the absence of a kick from gravitational recoil, has a mass of order the mass of the hole and an extent of order the hole's radius of influence,

$$
r_{\mathrm{BH}}=G M_{\mathrm{BH}} / \sigma_{*}^{2} .
$$

Throughout their dynamical evolution, these stellar clusters will lose mass by relaxation and expansion, as well as tidal disruptions by the black hole (Komossa \& Merritt 2008; O'Leary \& Loeb 2012). The relaxation timescale,

$$
t_{\mathrm{r}} \approx 10^{9}\left(\frac{M_{\mathrm{BH}}}{10^{5} M_{\odot}}\right)^{5 / 4} \mathrm{yr}
$$

ranges from several Myr for the smallest IMBHs to a Gyr for the largest. As IMBHs get accreted by the Milky Way early in the history of the Universe, the above processes can cause a $60-80 \%$ fractional loss of stellar mass by the present day (O'Leary \& Loeb 2012). At the same time, the physical extent of the clusters is expected to grow like $t^{1 / 3}$ after the first relaxation timescale (O'Leary \& Loeb 2012), resulting in a total expansion by a factor of 16 (for $\left.M_{\mathrm{BH}} \sim 10^{3} M_{\odot}\right)$ to 3 (for $\left.M_{\mathrm{BH}} \sim 5 \times 10^{4} M_{\odot}\right)$ over a Hubble time. Assuming that the initial extent of the clusters is dictated by the IMBH's sphere of influence at infall, their physical extent today would range between 0.5 and 1.0 pc. Despite their expansion, stellar clusters surrounding IMBHs would still be very compact today and may appear point-like or extended depending on their distance. Photometrically, they would have colors similar to those of old stars, while spectroscopically they would be distinguishable by the high velocity dispersions.

We have modelled the apparent brightness distribution of stellar cusps around naked IMBHs in the direct collapse scenario, which produces the largest number of massive IMBHs today. We assume an initial stellar mass that is twice the mass of the black hole and a present-day stellar mass-to-light ratio of 4 (as appropriate for an old stellar population with a Salpeter IMF see Rashkov et al. 2012, for details). The mass density in the star clusters is expected to follow a steep power law with drop-off power $\alpha \sim-2.15$. Given these physical characteristics, we calculate the peak surface brightness (within a typical SDSS seeing element of angular size $\sim 1$ arcsec) of the clusters as would be seen by a mock observer located $8 \mathrm{kpc}$ from the center of the VLII main halo. The distribution is shown in Figure 6. The angular size out to which the brightness profile will be brighter than $25 \mathrm{mag} \operatorname{arcsec}^{-2}$ is shown in Figure 7. Most clusters would be resolvable, as their angular extent spans 2 to 10 arcseconds. In these calculations, we have accounted for the fact that the SDSS points away from the Galactic Center by excluding IMBHs within $8 \mathrm{kpc}$ of 

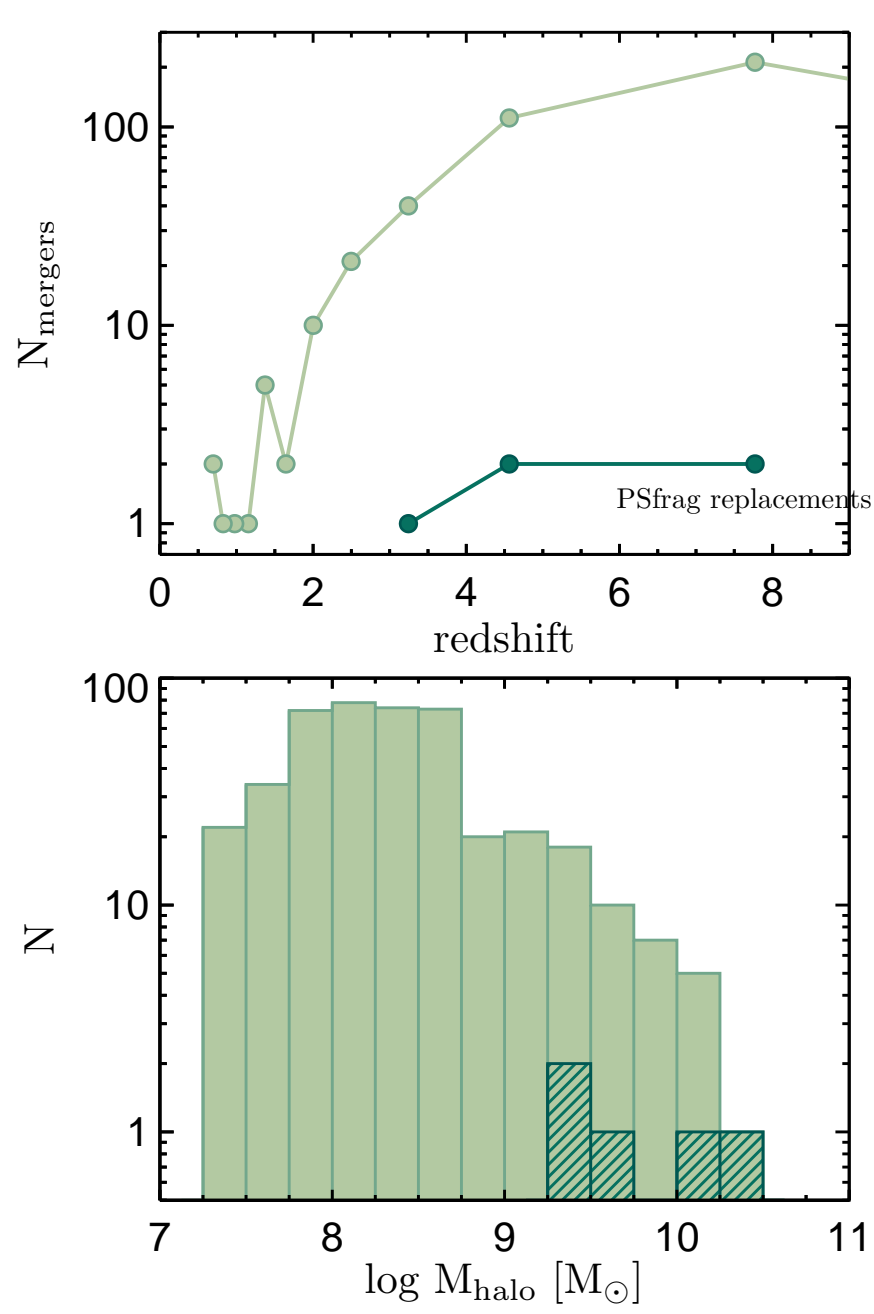

FIG. 4.- Top panel: Number of subhalo major mergers that produce a recoiling IMBH, as a function of redshift. Light green curve: Pop III remnants model. Dark green curve: Direct collapse precursors. Lower panel: Mass distribution (at infall) of all the subhalos whose central black holes were ejected following a major merger. The color scheme is the same as in the top panel.

the VLII halo center. The resulting distribution of peak brightness for the IMBH stellar clusters would have to be further scaled down by a factor of 5 , to account for the limited sky coverage of the SDSS. After accounting for these corrections, only a handful of clusters should be detectable with current surveys. This is consistent with a recent search for stellar clusters surrounding recoiled IMBHs in the SDSS source catalog that resulted in an upper limit of 100 candidates in the Milky Way halo (O'Learv \& Loeb 2012). We have also estimated the proper motion of IMBHs on the sky. Having tangential velocities ranging from 0 to $250 \mathrm{~km} \mathrm{~s}^{-1}$ as seen from an observer at $8 \mathrm{kpc}$ from the VLII halo center, and distances of up to $100 \mathrm{kpc}$, their proper motions fall in the range between 0.1 and 10 milliarcsec year ${ }^{-1}$. This is similar to measured proper motions of distant halo stars and may be currently detectable with the Hubble Space Telescope (Sohn et al. 2012), and future missions such as Gaia.

Finally, we assess the impact of a steeper $M_{\mathrm{BH}}-\sigma_{*}$ relation as measured by McConnell \& Ma (2013) in Figure 8. Like before, we tag all IMBHs according such relation and then remove from the final catalog all recoiling holes.

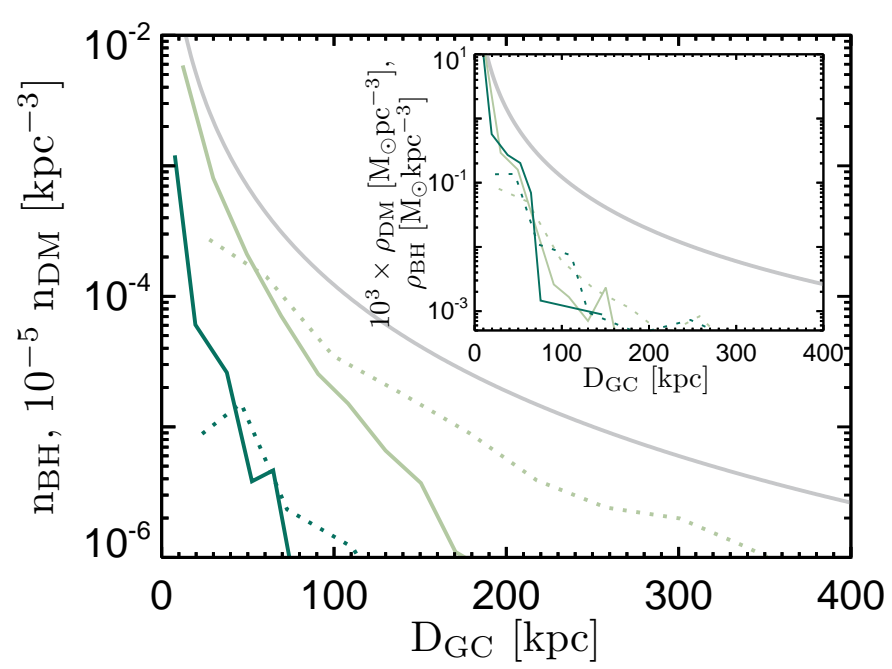

FIG. 5.- Spatial distribution of Galactic IMBHs versus the dark matter density profile in VLII. Main panel: IMBH number density Inset: IMBH mass density. The mass density of the dark matter has been multiplied by a factor of 1,000 for comparison. Light green: Pop III remnants scenario, naked (solid curves) and clothed (dotted curves) IMBHs. Dark green: direct collapse progenitors scenario, naked (solid curves) and clothed (dotted curves) IMBHs. Gray: VLII dark matter density profile. The total number density of IMBHs follows the dark matter density profile, while their mass density does not.

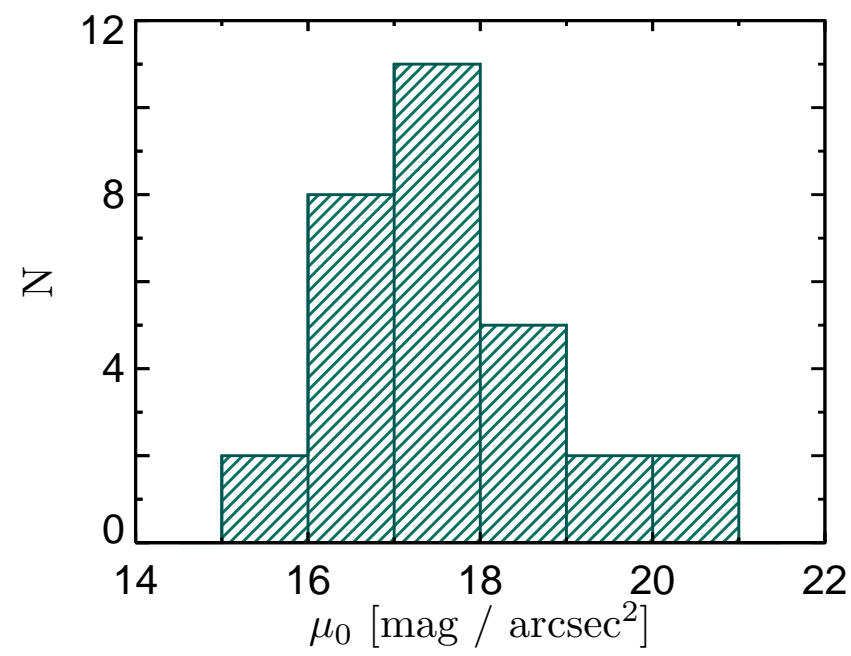

FIG. 6.- Distribution of V-band peak surface brightness (within the central 1 arcsec) of stellar cusps around naked IMBHs in the "direct collapse" model. Only $20 \%$ of the initial stellar mass (equal to $2 \times M_{\mathrm{BH}}$ ) is assumed to be retained today. To account for the partial SDSS sky coverage, the expected numbers should be scaled down by a factor of $\sim 5$.

The steeper slope (with exponent equal to 5.64 instead of 4) produces much lighter black holes, by about a factor of 30 , over the whole spectrum of masses. This effect is highlighted by the migration of most holes in the Pop III scenario to the lightest (leftmost) bin in the histogram in Figure 8. This $30 \times$ reduction in mass of the largest IMBHs results in a $30 \times$ reduction in the initial size of their stellar cusps, and in a large reduction of the stellar relaxation timescale, making them too compact and faint to be detectable with current surveys. A similar result would hold for those models in which the IMBHs hosted by dwarf satellites fall below the $M_{\mathrm{BH}}-\sigma_{*}$ relation, as in van Wassenhove et al. (2010). 


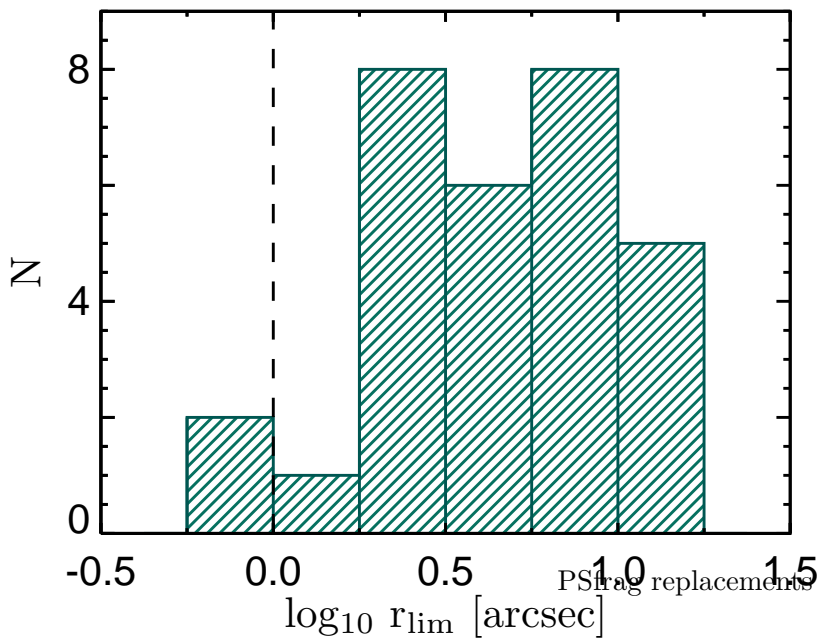

FIG. 7.- Angular size of stellar clusters around Galactic "naked" IMBHs in the "direct collapse" model (see text for details). The angular extent is calculated for an observer at $d_{\mathrm{GC}}=8 \mathrm{kpc}$ from the center of the VLII halo. Dashed line: angular resolution limit of current ground-based surveys.

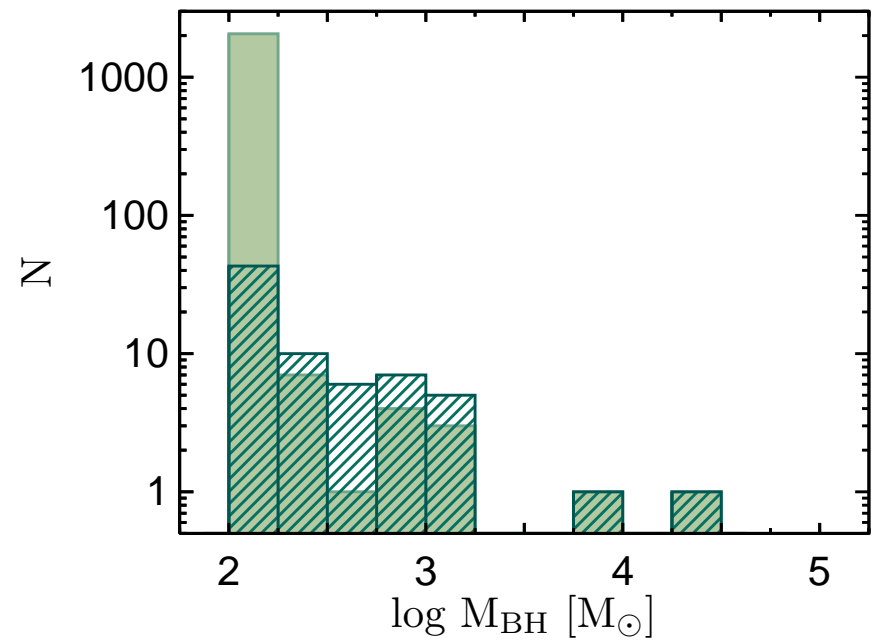

FIG. 8. - The impact of the steeper $M_{\mathrm{BH}}-\sigma_{*}$ relation from McConnell \& Ma (2013) on the mass function of relic IMBHs. Solid histogram: Pop III remnants. Hatched histogram: Direct collapse precursors. Comparison with Figure 3 clearly shows the significant reduction in the predicted masses of IMBHs.

We acknowledge useful discussions on the topics of this paper with Z. Haiman, S. Tremaine, and M. Volonteri. Support for this work was provided by the NSF through grant OIA-1124453, and by NASA through grant NNX12AF87G.

\section{REFERENCES}

Baker, J. G., Boggs, W. D., Centrella, J., et al. 2008, ApJ, 682, L29

Barth, A. J., Greene, J. E., \& Ho, L. C. 2005, ApJ, 619, L151

Begelman, M. C., Volonteri, M., \& Rees, M. J. 2006, MNRAS, 370, 289

Bellovary, J., Volonteri, M., Governato, F., et al. 2011, ApJ, 742, 13

Binney, J., \& Tremaine, S. 2008, Galactic Dynamics (Princeton: Princeton University Press)

Cooper, A. P., Cole, S., Frenk, C. S., et al. 2010, MNRAS, 406, 744

Devecchi, B., Volonteri, M., Rossi, E. M., Colpi, M., \& Portegies Zwart, S. 2012, MNRAS, 421, 1465

Diemand, J., Kuhlen, M., \& Madau, P. 2006, ApJ, 649, 1

Diemand, J., Kuhlen, M., Madau, P., et al. 2008, Nature, 454, 735

Di Matteo, T., Colberg, J., Springel, V., Hernquist, L., \& Sijacki, D. 2008, ApJ, 676, 33

Dubois, Y., Devriendt, J., Slyz, A., \& Teyssier, R. 2012, MNRAS, 420,2662

Fan, X. 2006, NewAR, 50, 665

Farrell, S. A., Webb, N. A., Barret, D., Godet, O., \& Rodrigues, J. M. 2009, Nature, 460, 73

Ferrarese, L., \& Merritt, D. 2000, ApJ, 539, L9

Gebhardt, K., Kormendy, J., Ho, L. C., et al. 2000, ApJ, 543, L5

Gebhardt, K., Rich, R. M., \& Ho, L. C. 2005, ApJ, 634, 1093

Ghez, A. M., Salim, S., Weinberg, N. N., et al. 2008, ApJ, 689, 1044

Graham, A. W., Onken, C. A., Athanassoula, E., \& Combes, F. 2011, MNRAS, 412, 2211

Guedes, J., Madau, P., Mayer, L., \& Callegari, S. 2011, ApJ, 729, 125

Haiman, Z. 2012, in The First Galaxies - Theoretical Predictions and Observational Clues, eds. T. Wiklind, V. Bromm, \& B. Mobasher, Springer ASSL, in press (arXiv:1203.6075

Häring, N., \& Rix, H.-W. 2004, ApJ, 604, L89

Inoue, K. T., Rashkov, V., Silk, J., \& Madau, P. 2013, arXiv:1301.5067

Islam, R. R., Taylor, J. E., \& Silk, J. 2003, MNRAS, 340, 647

Islam, R. R., Taylor, J. E., \& Silk, J. 2004, MNRAS, 354, 427

Kaaret, P., Prestwich, A. H., Zezas, A., et al. 2001, MNRAS, 321, L29
Kazantzidis, S., Mayer, L., Colpi, M., et al. 2005, ApJ, 623, L67 Komossa, S., \& Merritt, D. 2008, ApJ, 683, L21

Koposov, S. E., Yoo, J., Rix, H.-W., et al. 2009, ApJ, 696, 2179

Koushiappas, S. M., Bullock, J. S., \& Dekel, A. 2004, MNRAS, 354,292

Lodato, G., \& Natarajan, P. 2006, MNRAS, 371, 1813

Loeb, A., \& Rasio, F. A. 1994, ApJ, 432, 52

Lützgendorf, N., Kissler-Patig, M., Gebhardt, K., et al. 2012, arXiv: 1212.3475

Madau, P., \& Rees, M. J. 2001, ApJ, 551, L27

Mayer, L., Kazantzidis, S., Escala, A., \& Callegari, S. 2010, Nature, 466, 1082

McConnell, N. J., \& Ma, C.-P. 2013, ApJ, 764, 184

Merritt, D., Schnittman, J. D., \& Komossa, S. 2009, ApJ, 699, 1690

Micic, M., Abel, T., \& Sigurdsson, S. 2006, MNRAS, 372, 1540

Micic, M., Holley-Bockelmann, K., \& Sigurdsson, S. 2011, MNRAS, 414, 1127

Noyola, E., Gebhardt, K., Kissler-Patig, M., et al. 2010, ApJ, 719, L60

O'Leary, R. M., \& Loeb, A. 2009, MNRAS, 395, 781

O'Leary, R. M., \& Loeb, A. 2012, MNRAS, 421, 2737

Orosz, J. A., McClintock, J. E., Narayan, R., et al. 2007, Nature, 449,872

Rashkov, V., Madau, P., Kuhlen, M., \& Diemand, J. 2012, ApJ, 745,142

Reines, A. E., Greene, J. E., \& Geha, M. 2013, ApJ, 775, 116

Simon, J. D., Geha, M., Minor, Q. E., et al. 2011, ApJ, 733, 46

Sohn, S. T., Anderson, J., \& van der Marel, R. P. 2012, ApJ, 753,

Stacy, A., \& Bromm, V. 2012, arXiv:1211.1889

Stadel, J. G. 2001, Ph.D. Thesis, University of Washington

Tanaka, T., \& Haiman, Z. 2009, ApJ, 696, 1798

Tremaine, S., Gebhardt, K., Bender, R., et al. 2002, ApJ, 574, 740 van Wassenhove, S., Volonteri, M., Walker, M. G., \& Gair, J. R. 2010, MNRAS, 408, 1139

Vink, J. S., de Koter, A., \& Lamers, H. J. G. L. M. 2001, A\&A, 369,574

Volonteri, M., Haardt, F., \& Madau, P. 2003, ApJ, 582, 559

Volonteri, M., \& Perna, R. 2005, MNRAS, 358, 913 
Wolf, J., Martinez, G. D., Bullock, J. S., et al. 2010, MNRAS, 406, 1220
Xiao, T., Barth, A. J., Greene, J. E., et al. 2011, ApJ, 739, 28 